\title{
Three-dimensional quantum-optical input-output relations for arbitrary planar dielectrics
}

\author{
Salvatore Savasta, Omar Di Stefano, and Raffaello Girlanda \\ Istituto Nazionale per la Fisica della Materia and Dipartimento di Fisica della Materia e Tecnologie, Fisiche \\ Avanzate, Università di Messina, Salita Sperone 31, I-98166 Messina, Italy
}

\begin{abstract}
We present a three-dimensional quantum theory of light scattering, absorption, and emission in arbitrary planar dielectric systems. The theory is based on a scheme for quantization of the electromagnetic field in dispersive and lossy arbitrary planar dielectrics that takes explicitly into account the finite extent of the medium. We present general quantum-optical input-output relations of the output TE and TM photon operators to the corresponding input photon operators and to the reservoir noise operators. These three-dimensional inputoutput relations are uniquely determined by knowledge of the classical light modes of the planar dielectric structure.
\end{abstract}

\section{INTRODUCTION}

The analysis of those optical processes that take place inside or adjacent to material bodies, in which fluctuations in the electromagnetic vacuum and more generally the quantum statistical properties of light play a role, requires the development of a quantum theory of electrodynamics in media. Typical examples of such optical processes are spontaneous emission by an excited atom inside or adjacent to a material body, ${ }^{1-5}$ the Casimir effect, ${ }^{6}$ the quantum-state transformation of nonclassical light propagating through optical devices, ${ }^{7-9}$ the analysis of quantum noise in light-emitting devices. ${ }^{10}$ There is considerable interest in and research toward the development of quantization schemes in materials of increasingly general properties. Methods have been developed in the past years for quantization of the electromagnetic fields that display dispersion, absorption, and various degrees of inhomogeneity. The quantization of an electromagnetic field in absorptive dielectrics is complicated by the fact that the loss inevitably couples the field to a reservoir whose oscillators act as noise sources according to the fluctuation-dissipation theorem. A microscopically consistent description of quantized radiation in dispersive and absorptive linear bulk dielectrics was developed by Huttner and Barnett. ${ }^{11}$ They described the dielectric as a polarization field that interacts with both the light field and a continuum of reservoir fields to determine absorption, and they diagonalized the coupled radiation-matter Hamiltonian by a generalized Hopfield transformation. This method has been used to calculate the vacuum field fluctuations in absorbing dielectrics ${ }^{4}$ and hence to obtain the spontaneous-emission rate for an excited atom embedded in an infinitely absorbing dielectric medium. A widely adopted approach to quantization in lossy dielectrics uses Langevin forces to represent the noise ${ }^{12-15}$ and has been applied to the calculation of quantum-optical processes in dielectric slabs with local susceptibility ${ }^{12,16}$ and to the calculation of Casimir effects in absorbing dielectrics. ${ }^{17}$ This method has been applied to the analysis of the propagation of light pulses in slabs ${ }^{18}$ and of the propagation of nonclassical light in dielectrics. ${ }^{7,8}$ This quantization scheme has also been used for deriving normal-incidence quantum-optical input-output relations for general multilayer dielectric plates. ${ }^{9,19,20}$ These quantization schemes for lossy dielectrics with local dielectric permittivity were recently extended to the case of systems that display a nonlocal susceptibility. ${ }^{21}$

The quantized field expressions obtained for dielectric media with one or more interfaces were generally restricted to propagation in one dimension perpendicular to the dielectric surfaces, and these are adequate for a wide range of optical systems in which plane-parallel light beams are arranged to fall perpendicularly onto material bodies. A further range of observable effects, such as the spontaneous emission of atoms close to dielectric surfaces, vacuum field Casimir forces on dielectric surfaces, propagation of noncollinear twin beams, and propagation of light pulses through dielectric tunneling barriers, require a quantized field theory that incorporates propagation in all the spatial directions. Recently, Dung et al. ${ }^{22}$ and Scheel et al. ${ }^{23}$ presented a three-dimensional general quantization scheme for dispersive and absorbing inhomogeneous media based on the classic Green-tensor integral representation of the electromagnetic field. In particular, Scheel et al. have proved that the fundamental equal-time commutation relations of QED are preserved for an arbitrary space-dependent, Kramers-Krönigconsistent permittivity. Such three-dimensional quantization schemes, although they are quite general, do not explicitly treat the boundary of the inhomogeneous media with free space and thus do not describe explicitly the scattering and emission processes that are usually experimentally investigated. A different general threedimensional quantization scheme that makes use of a set of auxiliary fields and is followed by a canonical quantization procedure has been developed by Tip. ${ }^{24}$ Recently 
the equivalence of the quantization schemes of Scheel et $a l .{ }^{23}$ and Tip $^{24}$ has been demonstrated. ${ }^{25}$

In this paper we present quantum-optical input-output relations in dispersive and lossy bound and unbound inhomogeneous dielectrics that incorporate propagation in all the spatial directions. These relations allow us to analyze the propagation of the quantized radiation coming from free space toward the dielectric and then reflected back and to analyze the light emitted in free space by the dielectric, as happens in the usual kind of experiment. In this respect the present research can be considered a three-dimensional generalization of Refs. 13 and 19 and the generalization to arbitrary planar dielectrics of our recent paper ${ }^{26}$ in which we derived threedimensional input-output quantum-optical relations for a semi-infinite medium. The present treatment, in contrast to that of our earlier paper, ${ }^{26}$ allows us to obtain all the results without performing any explicit calculation based on the specific dielectric structure, thus providing a deep and general physical understanding of the scattering processes.

In the dielectric the electromagnetic field is inextricably coupled to the medium's polarization and hence to the reservoir; as a consequence it is not possible to define space-independent photon operators that obey the prescribed Bosonic commutation rules. In free space both the input and the output fields propagate freely, without interaction with the dielectric, and we show how it is possible to define naturally input and output photon operators in free space. In particular, we present useful and general input-output relations that relate the output TE and TM photon operators to the corresponding input photon operators and to the reservoir noise operators. We also verify that the output photon operators obtained obey the usual Bosonic commutation rules. These output photon operators are functions only of the corresponding classic light modes. The resultant operator expressions are easy to use, and they provide all the information that is needed for calculating the measurable properties of propagating as well as evanescent light fields in planar systems. If the quantum state of input radiation and of the material system is known, any output photon correlation can be directly calculated from these relations, provided that the classic light modes have been computed.

\section{PRELIMINARIES}

To quantize the electromagnetic field in absorbing dielectrics, we use position-dependent quantum operators in the Heisenberg picture. ${ }^{26}$ The frequency Fourier components for a generic position-dependent Heisenberg operator are defined as

$$
\hat{v}(\mathbf{r}, t)=\int_{-\infty}^{\infty} \mathrm{d} w \hat{v}(\mathbf{r}, \omega) \exp (-i \omega t) .
$$

As usual, the field operators can be separated into positive and negative frequency components $\hat{v}(\mathbf{r}, t)$ $=\hat{v}^{+}(\mathbf{r}, t)+\hat{v}^{-}(\mathbf{r}, t)$ where $\hat{v}^{-}(\mathbf{r}, t)=\left[\hat{v}^{+}(\mathbf{r}, t)\right]^{\dagger}$ and with Fourier-transform operators defined according to

$$
\hat{v}^{+}(\mathbf{r}, t)=\int_{0}^{\infty} \mathrm{d} \omega \hat{v}^{+}(\mathbf{r}, \omega) \exp (-i \omega t) .
$$

In this paper we consider the propagation of arbitrary polarized fields propagating in arbitrary directions through a planar medium with interfaces parallel to the $x-y$ plane. We split any incident transverse wave of arbitrary polarization into transverse electric (TE) and transverse magnetic (TM) components, and we exploit the translational symmetry in the $x-y$ directions by introducing the planar Fourier transform for the operators that describe the electromagnetic field and the dielectric. A generic operator can thus be expanded as

$$
\hat{\mathbf{v}}(\mathbf{r}, \omega)=\frac{1}{\sqrt{\mathcal{A}}} \sum_{\mathbf{K}} \exp (i \mathbf{K} \cdot \mathbf{R}) \hat{\mathbf{v}}_{\mathbf{K}}(z, \omega),
$$

where $\mathcal{A}$ is a quantization surface and we have indicated by $\mathbf{R}$ the in-plane position $[\mathbf{r}=(\mathbf{R}, z)]$. Of course $\hat{\mathbf{v}}_{\mathbf{K}}(z)$ can be further expanded in $k_{z}$ Fourier components, $\hat{\mathbf{v}}_{\mathbf{K}}(z)=(1 / \sqrt{\mathcal{L}}) \Sigma_{k_{z}} \hat{\mathbf{v}}_{\mathbf{k}} \exp \left(i k_{z} z\right)$ with $\mathbf{k}=\left(\mathbf{K}, k_{z}\right)$, where $\mathcal{V}=\mathcal{A L}$ is the quantization volume. In what follows, we also consider fields polarized along a given direction, which can be expanded as

$$
\hat{\mathbf{v}}(\mathbf{r})=\frac{1}{\sqrt{\mathcal{V}}} \sum_{\hat{\sigma}, \mathbf{k}} \exp (i \mathbf{k} \cdot \mathbf{r}) \widetilde{\mathbf{e}}_{\tilde{\sigma}, k} \hat{v}_{\tilde{\sigma}, k},
$$

where $\hat{e}_{\tilde{\sigma}, \mathbf{k}}$ are unit vectors that give the orthogonal polarization directions. In what follows, consider an orthogonal set of unit vectors that specify TE $(\widetilde{\sigma}=s)$, TM $(\widetilde{\sigma}$ $=p)$, and longitudinal $(L)(\widetilde{\sigma}=l)$ modes. We indicate by $\widetilde{e}_{\tilde{\sigma}, k}^{(i)} \equiv \widetilde{\mathbf{e}}_{\tilde{\sigma}, \mathbf{k}} \cdot \widetilde{\mathbf{x}}_{i}$ the $i$ th component of the polarization unit vectors, where $\mathbf{x}_{i}$ is the unit vector along axis $i$. We also use vectors of given in-plane wave vectors and polarization, $\hat{\mathbf{v}}_{\tilde{\sigma}, \mathbf{K}}(z)=(1 / \sqrt{\mathcal{L}}) \Sigma_{k_{z}} \widetilde{\mathbf{e}}_{\tilde{\sigma}, \mathbf{k}} \exp \left(i k_{z} z\right) \hat{v}_{\tilde{\sigma}, k}$.

\section{QUANTIZATION SCHEME}

Let us start the quantization scheme by writing the Maxwell equations for the electric- and magnetic-field operators. As is well known, from the Heisenberg equations of motion for the field variables we can obtain the Maxwell equations for the field operators. By assuming that the dielectric medium interacts with the light field only by means of its' polarization, we obtain

$$
\begin{array}{r}
\nabla \times \hat{\mathbf{E}}^{+}(\mathbf{r}, \omega)-i \omega \hat{\mathbf{B}}^{+}(\mathbf{r}, \omega)=0, \\
\nabla \times \hat{\mathbf{B}}^{+}(\mathbf{r}, \omega)-i \omega \mu_{0}\left[\varepsilon_{0} \hat{\mathbf{E}}^{+}(\mathbf{r}, \omega)+\hat{\mathbf{P}}^{+}(\mathbf{r}, \omega)\right]=0, \\
\nabla \cdot\left[\varepsilon_{0} \hat{\mathbf{E}}^{+}(\mathbf{r}, \omega)+\hat{\mathbf{P}}^{+}(\mathbf{r}, \omega)\right]=0, \\
\nabla \cdot \hat{\mathbf{B}}^{+}(\mathbf{r}, \omega)=0 .
\end{array}
$$

We consider a nonmagnetic and an isotropic medium. In this case the electromagnetic field interacts with the medium only by means of the electric polarization density, and the linear susceptibility can be assumed to be a scalar that does not depend on $\widetilde{\sigma}$. The positive frequency components of the polarization-density operator $\hat{P}^{+}(\mathbf{r}, \omega)$ of such a dielectric medium can be written as

$$
\begin{aligned}
\hat{\mathbf{P}}^{+}(\mathbf{r}, \omega)= & \varepsilon_{0} \chi(\mathbf{r}, \omega) \hat{\mathbf{E}}^{+}(\mathbf{r}, \omega) \\
& +i\left[\varepsilon_{0} \chi^{I}(\mathbf{r}, \omega) / \pi\right]^{1 / 2} \mathbf{f}^{+}(\mathbf{r}, \omega),
\end{aligned}
$$


where the noise operators obey the following commutation relations:

$$
\begin{aligned}
& {\left[\hat{f}_{i}^{+}(\mathbf{r}, \omega), \hat{f}_{j}^{-}\left(\mathbf{r}^{\prime}, \omega^{\prime}\right)\right]=\delta_{i, j} \delta\left(\mathbf{r}-\mathbf{r}^{\prime}\right) \delta\left(\omega-\omega^{\prime}\right),} \\
& {\left[\hat{f}_{i}^{ \pm}(\mathbf{r}, \omega), \hat{f}_{j}^{ \pm}\left(\mathbf{r}^{\prime}, \omega^{\prime}\right)\right]=0,}
\end{aligned}
$$

where $\chi(\mathbf{r}, \omega)=\chi^{R}\left[(\mathbf{r}, \omega)+i \chi^{I}(\mathbf{r}, \omega)\right.$ is the linear complex and position-dependent susceptibility of the medium. Equation (5) can be regarded as a phenomenological expression of the linear polarization operator, where $\chi(\mathbf{r}, \omega)$ is an arbitrary space-dependent, Kramers-Krönigconsistent susceptibility, ${ }^{13,22}$ or can be considered the result of a microscopic calculation, where the linear dielectric excitations are modeled by Boson fields interacting with the light field and the reservoir operators. ${ }^{21}$ For planar dielectric media it is useful to write the polarization-density operator in terms of planar Fourier components according to Eq. (3), as follows:

$$
\begin{aligned}
\hat{\mathbf{P}}^{+}(\mathbf{r}, \omega)= & \varepsilon_{0} \chi(\mathbf{r}, \omega) \hat{\mathbf{E}}^{+}(\mathbf{r}, \omega) \\
& +i\left[\varepsilon_{0} \chi^{I}(\mathbf{r}, \omega) / \pi\right]^{1 / 2} \hat{\mathbf{f}}^{+}(\mathbf{r}, \omega),
\end{aligned}
$$

where $\hat{\mathbf{f}}_{\mathbf{K}}^{+}(z, \omega)$ are the noise operators that obey the following commutation relations:

$$
\left[\hat{f}_{\mathbf{K}, i}^{+}(z, \omega), \hat{f}_{\mathbf{K}^{\prime}, j}^{-}\left(z^{\prime}, \omega^{\prime}\right)\right]=\delta_{\mathbf{K}, \mathbf{K}^{\prime}} \delta_{i, j} \delta\left(z-z^{\prime}\right) \delta\left(\omega-\omega^{\prime}\right),
$$

$\left[\hat{f}_{\mathbf{K}, i}^{ \pm}(z, \omega), \hat{f}_{\mathbf{K}^{\prime}, j}^{ \pm}\left(z^{\prime}, \omega^{\prime}\right)\right]=0$

Inserting Eq. (5) into the Maxwell equations for the electromagnetic-field operators, we obtain the Maxwell equations for the dielectric medium, with a complex linear susceptibility and with a noise source term, that are the starting point for field quantization inside dielectric media. $^{22}$ Equations (4) and (5) imply that the electric field obeys the partial differential equation

$$
\left[-\nabla \times \nabla \times+\frac{\omega^{2}}{c^{2}} \varepsilon(\mathbf{r}, \omega)\right] \hat{\mathbf{E}}(\mathbf{r}, \omega)=-i \omega \mu_{0} \hat{\mathbf{j}}^{+}(\mathbf{r}, \omega),
$$

where $\hat{\mathbf{j}}(\mathbf{r}, \omega)$ is the noise current, given by

$$
\hat{\mathbf{j}}^{+}(\mathbf{r}, \omega)=\frac{\omega}{\mu_{0} c^{2}}\left[\frac{\hbar}{\varepsilon_{0} \pi} \chi^{\mathcal{I}}(\mathbf{r}, \omega)\right]^{1 / 2} \hat{\mathbf{f}}(\mathbf{r}, \omega) .
$$

The most general solution of Eq. (11) can be written as

$$
\begin{aligned}
\hat{\mathbf{E}}^{+}(\mathbf{r}, \omega)= & i \omega \hat{\mathbf{A}}_{h}^{+}(\mathbf{r}, \omega)+i \omega \mu_{0} \int \mathrm{d}^{3} r^{\prime} \mathbf{G}\left(\mathbf{r}, \mathbf{r}^{\prime}\right) \\
& \cdot \hat{\mathbf{j}}^{+}\left(\mathbf{r}^{\prime}, \omega\right),
\end{aligned}
$$

where $i \omega \hat{\mathbf{A}}_{h}^{+}(\mathbf{r}, \omega)$ is the homogeneous solution of Eq. (11) that describes the electromagnetic field in the absence of the noise currents and $\mathbf{G}\left(\mathbf{r}, \mathbf{r}^{\prime}\right)$ is the dyadic Green function of the classical problem. It satisfies the equation

$$
\left[-\nabla \times \nabla \times+\frac{\omega^{2}}{c^{2}} \varepsilon(\mathbf{r}, \omega)\right] \mathbf{G}^{\prime}\left(\mathbf{r}, \mathbf{r}^{\prime}, \omega\right)=\mathbf{1} \delta\left(\mathbf{r}-\mathbf{r}^{\prime}\right),
$$

where $\mathbf{1}$ is the unit tensor. If the absorbing medium is unbounded in all the spatial directions, the homogeneous term in Eq. (13) makes contribution. ${ }^{21}$ The contribution that is due to the homogeneous solution is further described in Section 5 below.

\section{LIGHT MODES AND THE GREEN FUNCTION IN PLANAR DIELECTRIC SYSTEMS}

In this section we consider only classic fields, and much of the material presented here consists of well-known results from the study of thin-film optics. We present it here for completeness and to introduce some results and notation that will be useful in Section 5. We analyze the propagation of light in a generic planar and isotropic dielectric system. The Maxwell equations are

$$
\begin{aligned}
\nabla \times \mathbf{E}(\mathbf{r}, \omega)-i \omega \mathbf{B}(\mathbf{r}, \omega) & =0, \\
\nabla \times \mathbf{B}^{+}(\mathbf{r}, \omega)-i \omega \mu_{0}\left[\varepsilon_{0} \mathbf{E}(\mathbf{r}, \omega)+\mathbf{P}(\mathbf{r}, \omega)\right] & =0 \\
\left.\nabla \cdot \varepsilon_{0} \mathbf{E}(\mathbf{r}, \omega)+\mathbf{P}(\mathbf{r}, \omega)\right] & =0, \\
\nabla \cdot \mathbf{B}(\mathbf{r}, \omega) & =0 .
\end{aligned}
$$

We can obtain the classic polarization density by taking the expectation value of Eq. (5), which is

$$
\mathbf{P}(\mathbf{r}, \omega)=\varepsilon_{0} \chi(\mathbf{r}, \omega) \mathbf{E}(\mathbf{r}, \omega) .
$$

By inserting Eq. (16) into Eq. (15), we obtain a linear and homogeneous system of differential equations. The general solution that satisfies this linear system of partial differential equations and also satisfies the boundary conditions at the interfaces and at infinity can be written as

$$
\begin{aligned}
\mathbf{E}(\mathbf{r}, \omega)= & \sum_{\sigma, \mathbf{K}} \exp (i \mathbf{K} \cdot \mathbf{R})\left[C_{\rangle, \sigma, \mathbf{K}} \mathbf{U}_{\sigma, \mathbf{K}}^{\rangle}(z, \omega)\right. \\
& +C_{\langle, \sigma, \mathbf{K}} \mathbf{U}_{\sigma, \mathbf{K}}^{\curlywedge}(z, \omega),
\end{aligned}
$$

where $\sigma=s, p$ specifies the polarization and $\rangle$ and $\langle$ indicate, respectively, rightward and leftward propagating modes of the field. All possible modes of the planar dielectric system are spanned by the coefficients $C_{\rangle /, \sigma, \mathbf{K}}$. There are thus four degrees of freedom for a given frequency component and a fixed in-plane wave vector that correspond to two possible transverse polarizations and to the leftward and rightward propagating input fields.

We consider in particular a planar dielectric structure embedded in free space. The whole space is divided into three regions: the left vacuum, the dielectric black box, and the right vacuum. We indicate rightward and leftward wave vectors for waves propagating in free space as $\mathbf{v}_{0, \pm}=\left(\mathbf{K}, \pm w_{0}\right)$, with $v_{0}^{2} \equiv \mathbf{v}_{0, \pm} \cdot \mathbf{v}_{0, \pm}=\omega^{2} / c^{2}$. Furthermore, the unit vectors $\widetilde{\mathbf{s}}$ and $\widetilde{\mathbf{p}}_{ \pm}$will span the possible orientations of $\mathbf{E}$. Vector $\overline{\mathbf{s}}=(\mathbf{K} / K) \times \overline{\mathbf{z}}$ is the unit vector for TE modes, whereas $\widetilde{\mathbf{p}}_{ \pm}=\left(K \widetilde{\mathbf{z}} \mp w_{0} \mathbf{K} / K\right) / v_{0}$ are the unit vectors for rightward and leftward propagating TM modes, respectively. In the two vacuum regions the rightward and the leftward light modes can be written as 


$$
\mathbf{U}_{\sigma, \mathbf{K}}^{\rangle}(z, \omega)=\exp \left(i w_{0} z\right) \bar{\eta}_{\sigma, 0}^{\rangle}+\exp \left(-i w_{0} z\right) r_{\sigma}^{\rangle} \bar{\eta}_{\sigma, 0}^{\langle}
$$

(in the left vacuum),

$$
\mathbf{U}_{\sigma, \mathbf{K}}^{\rangle}(z, \omega)=t_{\sigma} \exp \left(i w_{0} z\right) \widetilde{\eta}_{\sigma, 0}^{\rangle}
$$

(in the right vacuum),

$$
\mathbf{U}_{\sigma, \mathbf{K}}^{\langle}(z, \omega)=t_{\sigma} \exp \left(-i w_{0} z\right) \widetilde{\eta}_{\sigma, 0}^{\curlywedge}
$$

(in the left vacuum),

$$
\mathbf{U}_{\sigma, \mathbf{K}}^{\backslash}(z, \omega)=\exp \left(-i w_{0} z\right) \bar{\eta}_{\sigma, 0}^{\backslash}+\exp \left(i w_{0} z\right) r_{\sigma}^{\langle} \widetilde{\eta}_{\sigma, 0}
$$

(in the right vacuum),

where $\tilde{\eta}$ labels the polarization direction: $\tilde{\eta}_{s}^{\rangle /\langle}=\widetilde{\mathbf{s}}$ and $\tilde{\eta}_{p}^{\rangle / K}=\tilde{\mathbf{p}}_{ \pm} . \quad$ The orientations of the light modes $\mathbf{U}_{\sigma, \mathbf{K}}(z, \omega)$ are shown schematically in Fig. 1 . We have not indicated explicitly the dependence of the reflection and transmission coefficients on $\mathbf{K}$ and $\omega$. Of course these coefficients depend on the dielectric permittivity $\varepsilon(z)$ of the planar medium. Furthermore, we have introduced the general property that $t_{\sigma}^{\rangle}=t_{\sigma}^{\langle}$. This property originates from reciprocity, and we use it explicitly in Section 5 below. ${ }^{27,28}$ The light modes inside the black box and the reflection and transmission coefficients of the whole structure can easily be determined for layered systems, i.e., the planar dielectric structures made from homogeneous layers with the dielectric permittivity that is constant inside each layer, by use of the method of $2 \times 2$ transfer matrices. ${ }^{27-29}$ One can use this method to obtain the amplitude of electric and magnetic fields of any isotropic layered medium.

We have briefly analyzed the solution of the Maxwell homogeneous equations in a bounded layered dielectric. To determine the electric-field operator [see Eq. (13)], we are also interested to the fields that result from a specific source current. This case can be conveniently handled by standard dyadic Green-function methods. The Green dyadic satisfies Eq. (14), which can also be expressed in components as

$$
\begin{array}{r}
\sum_{\mu}\left[-\frac{\partial^{2}}{\partial r_{\mu} \partial r_{\lambda}}+\nabla^{2} \delta_{\lambda \mu}+\frac{w^{2}}{c^{2}} \delta_{\lambda \mu} \epsilon(z, \omega)\right] G_{\mu \nu}\left(\mathbf{r}, \mathbf{r}^{\prime}, \omega\right) \\
=\delta_{\lambda \nu} \delta\left(\mathbf{r}-\mathbf{r}^{\prime}\right)
\end{array}
$$

We can take advantage of the planar geometry by introducing the Green dyadic of given in-plane wave vector:

$$
\mathbf{G}\left(\mathbf{r}, \mathbf{r}^{\prime}, \omega\right)=(1 / \mathcal{A}) \sum_{\mathbf{K}} \exp \left[i \mathbf{K} \cdot\left(\mathbf{R}-\mathbf{R}^{\prime}\right)\right] \mathbf{G}_{\mathbf{K}}\left(z, z^{\prime}, \omega\right)
$$

Following Refs. 29 and 30, it is possible to write the components of the Green dyadic in terms of the light modes of the planar system. Choosing the plane $x-z$ as the plane of incidence, we can write the components of the Green dyadic as

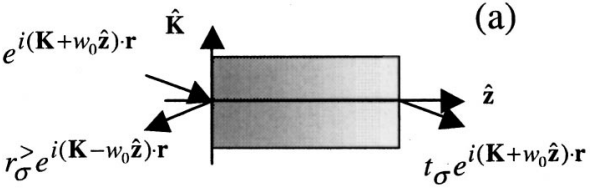

(b)

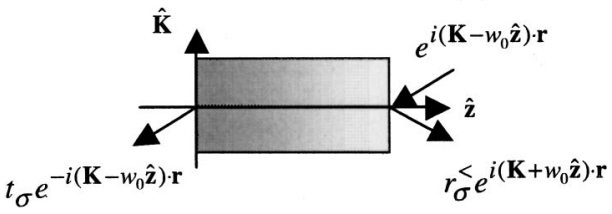

Fig. 1. Schematic representation of the light modes in the regions external to a bounded planar dielectric structure: (a) leftward modes, (b) rightward modes.

$G_{\mathbf{K}, \mathbf{x}, \mathbf{x}}\left(z, z^{\prime}, \omega\right.$

$$
\begin{aligned}
& =\frac{-1}{W_{p, \mathbf{K}}(\omega)}\left[U_{x, \mathbf{K}}^{\rangle}(z, \omega) U_{x, \mathbf{K}}^{\curlywedge}\left(z^{\prime}, \omega\right) \Theta\left(z-z^{\prime}\right)\right. \\
& \left.\quad+U_{x, \mathbf{K}}^{\backslash}(z, \omega) U_{x, \mathbf{K}}^{\rangle}\left(z^{\prime}, \omega\right) \Theta\left(z^{\prime}-z\right)\right],
\end{aligned}
$$

$G_{\mathbf{K}, \mathbf{z}, \mathbf{x}}\left(z, z^{\prime}, \omega\right)$

$$
\begin{aligned}
& =\frac{-1}{W_{p, \mathbf{K}}(\omega)}\left[U_{z, \mathbf{K}}^{\rangle}(z, \omega) U_{x, \mathbf{K}}^{\curlywedge}\left(z^{\prime}, \omega\right) \Theta\left(z-z^{\prime}\right)\right. \\
& \left.\quad+U_{z, \mathbf{K}}^{\langle}(z, \omega) U_{x, \mathbf{K}}^{\rangle}\left(z^{\prime}, \omega\right) \Theta\left(z^{\prime}-z\right)\right],
\end{aligned}
$$

$G_{\mathbf{K}, \mathbf{x}, \mathbf{z}}\left(z, z^{\prime}, \omega\right)$

$$
\begin{aligned}
& =\frac{1}{W_{p, \mathbf{K}}(\omega)}\left[U_{x, \mathbf{K}}^{\rangle}(z, \omega) U_{z, \mathbf{K}}^{\langle}\left(z^{\prime}, \omega\right) \Theta\left(z-z^{\prime}\right)\right. \\
& \left.\quad+U_{z, \mathbf{K}}^{\langle}(z, \omega) U_{z, \mathbf{K}}^{\rangle}\left(z^{\prime}, \omega\right) \Theta\left(z^{\prime}-z\right)\right],
\end{aligned}
$$

$G_{\mathbf{K}, \mathbf{z}, \mathbf{z}}\left(z, z^{\prime}, \omega\right)$

$$
\begin{aligned}
& =\frac{1}{W_{p, \mathbf{K}}(\omega)}\left[U_{z, \mathbf{K}}^{\rangle}(z, \omega) U_{z, \mathbf{K}}^{\langle}\left(z^{\prime}, \omega\right) \Theta\left(z-z^{\prime}\right)\right. \\
& \left.+U_{z, \mathbf{K}}^{\langle}(z, \omega) U_{z, \mathbf{K}}^{\rangle}\left(z^{\prime}, \omega\right) \Theta\left(z^{\prime}-z\right)\right] \\
& \quad+\frac{c^{2} \delta\left(z-z^{\prime}\right)}{\omega^{2} \epsilon\left(z^{\prime}, \omega\right)}
\end{aligned}
$$

$G_{\mathbf{K}, \mathbf{y}, \mathbf{y}}\left(z, z^{\prime}, \omega\right)$

$$
\begin{aligned}
= & \frac{1}{W_{s, \mathbf{K}}(\omega)}\left[U_{y, \mathbf{K}}^{\rangle}(z, \omega) U_{y, \mathbf{K}}^{\langle}\left(z^{\prime}, \omega\right) \Theta\left(z-z^{\prime}\right)\right. \\
& \left.+U_{y, \mathbf{K}}^{\langle}(z, \omega) U_{y, \mathbf{K}}^{\langle}\left(z^{\prime}, \omega\right) \Theta\left(z^{\prime}-z\right)\right], \quad
\end{aligned}
$$

where we have defined the components of the light modes as

$$
U_{\mathbf{K}, x_{i}}^{\backslash /}(z, \omega)=\sum_{\sigma} \mathbf{U}_{\sigma, \mathbf{K}}^{\rangle /}(z, \omega) \cdot \hat{\mathbf{x}}_{i} .
$$

The Wronskians $W_{\mathbf{K}}^{\sigma}(\omega)$ do not depend on $z$ and are given by

$$
\begin{aligned}
W_{s, \mathbf{K}}(\omega) & =W_{y y, \mathbf{K}}(\omega), \\
W_{p, \mathbf{K}}(\omega) & =W_{z z, \mathbf{K}}(\omega)-W_{x x, \mathbf{K}}(\omega),
\end{aligned}
$$

where 


$$
\begin{aligned}
W_{j j, \mathbf{K}}(\omega)= & U_{j, \mathbf{K}}^{\langle}(z, \omega) \frac{\partial}{\partial z} U_{j, \mathbf{K}}^{\rangle}(z, \omega) \\
& -U_{j, \mathbf{K}}^{\rangle}(z, \omega) \frac{\partial}{\partial z} U_{j, \mathbf{K}}^{\langle}(z, \omega) .
\end{aligned}
$$

The remaining components of the Green dyadic $\left(G_{\mathbf{K}, \mathbf{x}, \mathbf{y}}, G_{\mathbf{K}, \mathbf{y}, \mathbf{x}}, G_{\mathbf{K}, \mathbf{y}, \mathbf{z}}, G_{\mathbf{K}, \mathbf{z}, \mathbf{y}}\right)$ obey homogeneous equations and thus vanish identically. ${ }^{30}$ We point out that this mode expansion of the Green dyadic holds not only for layered media but for arbitrary planar dielectrics. The possibility of expanding the Green dyadic in terms of the light modes of the dielectric system can be useful, and, as we shall see in Sections 5 and 6, it lies at the basis of our quantization scheme and at the basis of the quantum-optical input-output relations for arbitrary planar dielectrics.

\section{FROM THE LIGHT MODES TO THE PHOTON OPERATORS}

In Section 2 we wrote the general electric-field operator of a given positive frequency [Eq. (13)] for an arbitrary absorbing linear dielectric system. Here we specialize to arbitrary planar systems, and, using the results shown in Section 3, we derive quantum-optical input-output relations for arbitrary planar dielectrics. According to Eq. (13), the general electric-field operator at a given positive frequency can be written as

$$
\hat{\mathbf{E}}^{+}(\mathbf{r}, \omega)=i \omega \hat{\mathbf{A}}_{h}^{+}(\mathbf{r}, \omega)+i \omega \mu_{0} \int \mathrm{d}^{3} r^{\prime} \mathbf{G}\left(\mathbf{r}, \mathbf{r}^{\prime}\right) \cdot \hat{\mathbf{j}}\left(\mathbf{r}^{\prime}, \omega\right) .
$$

According to what has been shown in Section 4, the homogeneous solution of Eq. (11) can be written as

$$
\begin{aligned}
\hat{\mathbf{A}}_{h}^{+}(\mathbf{r}, \omega)= & \sum_{\sigma, \mathbf{K}} \exp (i \mathbf{K} \cdot \mathbf{R})\left[\hat{C}_{\sigma, \mathbf{K}}^{\rangle}(\omega) \mathbf{U}_{\sigma, \mathbf{K}}^{\rangle}(z, \omega)\right. \\
& +\hat{C}_{\sigma, \mathbf{K}}^{\langle}(\omega) \mathbf{U}_{\sigma, \mathbf{K}}^{\langle}(z, \omega) .
\end{aligned}
$$

The unknown operators $\hat{C}_{s, \mathbf{K}}^{\rangle / /}$and $\hat{C}_{p, \mathbf{K}}^{\rangle /}$, as for the classic theory, are related to the degrees of freedom of the system. In what follows, we consider a planar dielectric system of finite extent embedded in free space. Treating such media of finite extent, we are able to analyze the propagation of the quantized radiation coming from free space toward the dielectric and reflected back and to analyze the light emitted in free space by the dielectric, as happens in the usual kinds of experiments. The less realistic semi-infinite and infinite planar structures can be treated analogously. ${ }^{21}$ We can determine the physical meaning of the unknown operators $\hat{C}_{s, \mathbf{K}}^{\rangle /\langle}$and $\hat{C}_{p, \mathbf{K}}^{\rangle /}$by looking at Eq. (24) in the two vacuum regions, on the left and on the right, of the planar dielectric system. ${ }^{21,26}$ Let us consider the electric-field operator in the left vacuum. For a given $\mathbf{K}$, the part of the electric field operator in Eq. (24) that describes input rightward waves coming from $-\infty$ is

$$
i \omega\left[\hat{C}_{s, \mathbf{K}}^{\rangle}(\omega) \exp \left(i w_{1} z\right) \widetilde{\mathbf{s}}+\hat{C}_{p, \mathbf{K}}^{\rangle}(\omega) \exp \left(i w_{1} z\right) \widetilde{\mathbf{p}}_{1+}\right] .
$$

As required by causality, and as can be seen explicitly by use of Eq. (21), the term that is due to the particular solution does not produce rightward waves in the left vacuum. We observe that rightward waves in free space coming from $-\infty$ cannot sense the presence of the interface and of the dielectric medium before these waves arrive in the region occupied by the black box, and thus they have the same properties as the corresponding quantum operators in free space. An identical argument holds in the rightward vacuum for the input leftward waves coming from $+\infty$. Following the usual quantization scheme in free space, we find that

$$
\hat{C}_{s(p), \mathbf{K}}^{\rangle /\langle}(\omega)=\alpha_{\mathbf{K}}(\omega) \hat{a}_{s(p), \mathbf{K}}^{\rangle /\langle}(\omega),
$$

with $\alpha_{\mathbf{K}}(\omega)=\left(\hbar / 4 \pi \varepsilon_{0} c^{2} w_{1}\right)^{1 / 2}$. The operators $\hat{a}_{\sigma, \mathbf{K}}^{\tau}(\omega)$ are vacuum photon operators that obey the usual Bose commutation rules $(\tau=\rangle,\langle)$ :

$$
\begin{aligned}
& {\left[\hat{a}_{\sigma, \mathbf{K}}^{\tau}(\omega), \hat{a}_{\sigma^{\prime}, \mathbf{K}^{\prime}}^{\tau^{\prime} \dagger}\left(\omega^{\prime}\right)\right]=\delta_{\mathbf{K}, \mathbf{K}^{\prime}} \delta_{\sigma, \sigma^{\prime}} \delta_{\tau, \tau^{\prime}} \delta\left(\omega-\omega^{\prime}\right),} \\
& {\left[\hat{a}_{\sigma, \mathbf{K}}^{\tau}(\omega), \hat{a}_{\sigma^{\prime}, \mathbf{K}^{\prime}}^{\tau^{\prime}}\left(\omega^{\prime}\right)\right]=0 .}
\end{aligned}
$$

We have thus specified the unknown coefficients, and the quantization procedure is now complete. By using Eq. (20) we can write the total quantized electric field as

$$
\hat{\mathbf{E}}(\mathbf{r}, t)=\hat{\mathbf{E}}^{+}(\mathbf{r}, t)+\hat{\mathbf{E}}^{-}(\mathbf{r}, t),
$$

with the positive electric field operator given by

$$
\hat{\mathbf{E}}^{+}(\mathbf{r}, t)=\int_{0}^{\infty} \mathrm{d} \omega \exp (-i \omega t) \hat{\mathbf{E}}^{+}(\mathbf{r}, \omega)
$$

and the positive frequency components of the electric field operator given by

$$
\hat{\mathbf{E}}^{+}(\mathbf{r}, \omega)=\frac{1}{\sqrt{\mathcal{A}}} \sum_{\mathbf{K}} \exp (i \mathbf{K} \cdot \mathbf{R}) \hat{\mathbf{E}}_{\mathbf{K}}^{+}(z, \omega),
$$

with

$$
\begin{aligned}
\hat{\mathbf{E}}_{\mathbf{K}}^{+}(z, \omega)= & i \omega \alpha_{\mathbf{K}}(\omega) \sum_{\tau, \sigma} \mathbf{U}_{\sigma, \mathbf{K}}^{\tau}(z, \omega) \hat{a}_{\sigma, \mathbf{K}}^{\tau} \\
& +i \omega \mu_{0} \int_{-\infty}^{\infty} \mathrm{d} z^{\prime} \mathbf{G}_{\mathbf{K}}\left(z, z^{\prime}, \omega\right) \cdot \hat{\mathbf{j}}_{\mathbf{K}}\left(z^{\prime}, \omega\right)
\end{aligned}
$$

This expression for the positive frequency components of the electric-field operator is valid both in free space and in the medium. The corresponding expressions for the other field variables of interest can be derived directly from knowledge of the components $\hat{\mathbf{E}}^{+}(\mathbf{r}, \omega)$ of the electric-field operator. ${ }^{22}$

By explicitly using mode expansion (21) of the Green dyadic, it is possible to write the electric-field operator in the leftward vacuum as 


$$
\begin{aligned}
\hat{\mathbf{E}}_{\mathbf{K}}^{+}(z, \omega)= & i \omega \alpha_{\mathbf{K}}(\omega)\left[\hat{a}_{s, \mathbf{K}}^{\rangle}(\omega) \widetilde{\mathbf{s}} \exp \left(i w_{1} z\right)\right. \\
& +\hat{b}_{s, \mathbf{K}}^{\backslash}(\omega) \widetilde{\mathbf{s}} \exp \left(-i w_{1} z\right) \\
& +\hat{a}_{p, \mathbf{K}}^{\rangle}(\omega) \widetilde{\mathbf{p}}_{1+} \exp \left(i w_{1} z\right) \\
& \left.+\hat{b}_{p, \mathbf{K}}^{\langle}(\omega) \widetilde{\mathbf{p}}_{1-} \exp \left(-i w_{1} z\right)\right]
\end{aligned}
$$

where the leftward output operators $\hat{b}_{\sigma, \mathbf{K}}^{<}(\omega)$, which describe light escaping from the dielectric at the left interface with free space, are given by

$$
\hat{b}_{\sigma, \mathbf{K}}^{\langle}(\omega)=r_{\sigma}^{\rangle} \hat{a}_{\sigma, \mathbf{K}}^{\rangle}(\omega)+t_{\sigma} \hat{a}_{\sigma, \mathbf{K}}^{\langle}(\omega)+F_{\mathbf{K}, \sigma}^{\rangle}(\omega),
$$

where

$$
F_{\mathbf{K}, \sigma}^{\tau}(\omega)=\frac{\mu_{0}}{2 i w_{0} \alpha_{\mathbf{K}}(\omega)} \int_{-\infty}^{\infty} \mathrm{d} z^{\prime} \tilde{\mathbf{U}}_{\sigma, \mathbf{K}}^{\tau}\left(z^{\prime}, \omega\right) \cdot \hat{\mathbf{j}}_{\mathbf{K}}\left(z^{\prime}, \omega\right) .
$$

We have defined $\tilde{\mathbf{U}}_{p, \mathbf{K}}^{\tau} \equiv\left(-U_{x, \mathbf{K}}^{\tau}, U_{y, \mathbf{K}}^{\tau}, U_{z, \mathbf{K}}^{\tau}\right)$ and $\tilde{\mathbf{U}}_{s, \mathbf{K}}^{\tau}$ $\equiv \mathbf{U}_{s, \mathbf{K}}^{\tau}$. Equation (35) is a quantum-optical inputoutput relation for absorbing arbitrary planar dielectrics. Equation (35) holds also for absorbing media that are bounded only at the left side. In this case we have only to drop the term $t^{\sigma} \hat{a}_{\sigma, \mathbf{K}}^{\langle}(\omega)$, because in this case the leftward input is always absorbed before it can reach the interface. The operators $F_{\mathbf{K}, \sigma}^{\tau}(\omega)$ obey simple commutation relations, which are derived in Section 6 below. In particular, we shall find that

$$
\begin{aligned}
{\left[F_{\mathbf{K}, \sigma}^{\tau}(\omega), F_{\mathbf{K}^{\prime}}^{\tau, \dagger}\left(\omega^{\prime}\right)\right]=} & \delta\left(\omega-\omega^{\prime}\right) \\
& \times \delta_{\mathbf{K}, \mathbf{K}^{\prime}} \delta_{\sigma, \sigma^{\prime}}\left(1-\left|r_{\sigma}^{\tau}\right|^{2}-\left|t_{\sigma}\right|^{2}\right) .
\end{aligned}
$$

Thus this commutator is associated with the total absorption of the corresponding mode inside the dielectric structure.

From Eq. (35) we can recover the results for the semiinfinite absorbing dielectric. ${ }^{26}$ Analogously, the components of the electric-field operator in the leftward vacuum can be written as

$$
\begin{aligned}
\hat{\mathbf{E}}_{\mathbf{K}}^{+}(\mathbf{z}, \omega)= & i \omega \alpha_{\mathbf{K}}(\omega)\left[\hat{a}_{s, \mathbf{K}}^{\langle}(\omega) \widetilde{\mathbf{s}} \exp \left(-i w_{0} z\right)\right. \\
& +\hat{b}_{s, \mathbf{K}}^{\rangle}(\omega) \widetilde{\mathbf{s}} \exp \left(i w_{0} z\right) \\
& +\hat{a}_{p, \mathbf{K}}^{\backslash}(\omega) \widetilde{\mathbf{p}}_{1-} \exp \left(-i w_{0} z\right) \\
& \left.+\hat{b}_{p, \mathbf{K}}^{\rangle}(\omega) \widetilde{\mathbf{p}}_{1+} \exp \left(i w_{0} z\right)\right],
\end{aligned}
$$

where the rightward output operators $\hat{b}_{\sigma, \mathbf{K}}^{\rangle}(\omega)$ are given by

$$
\hat{b}_{\sigma, \mathbf{K}}^{\rangle}(\omega)=r_{\sigma}^{\langle} \hat{a}_{\sigma, \mathbf{K}}^{\langle}(\omega)+t_{\sigma} \hat{a}_{\sigma, \mathbf{K}}(\omega)+F_{\mathbf{K}, \sigma}^{\langle}(\omega) .
$$

Equations (35) and (38) are general three-dimensional quantum-optical input-output relations (see scheme in Fig. 2) that relate the output field operators to the input photon operators and to the noise reservoir operators. We note that, despite the fact that the noise reservoir operators are vectors, it has been possible to define scalar output operators in complete analogy with the input photon operators. These quantum-optical input-output relations are general and simple to use for particular needs.

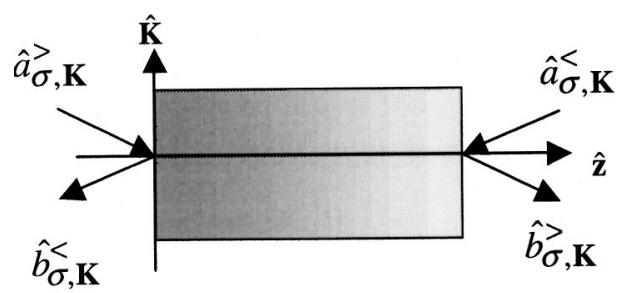

Fig. 2. Notation for the annihilation operators used in the three-dimensional quantum-optical input-output relations.

It is sufficient to know the classic light modes of the planar dielectric system to obtain the specific required input-output relation.

We can perform a mode decomposition of the electricfield operator inside the planar dielectric also. We do this by introducing mode expansion (21) of the Green dyadic into Eq. (33). In this case the resultant operators are no longer position independent. We obtain

$$
\begin{aligned}
\mathbf{E}_{\mathbf{K}}^{+}(z, \omega)= & i \omega \mu_{0} \frac{c^{2}}{\omega^{2} \epsilon(z, \omega)} \hat{j}_{z}(z, \omega) \widetilde{\mathbf{z}} \\
& +i \omega \alpha_{\mathbf{K}}(\omega)\left[\mathbf{U}_{\sigma, \mathbf{K}}^{\rangle}(z, \omega) c_{\sigma, \mathbf{K}}\right\rangle(z, \omega) \\
& \left.+\mathbf{U}_{\sigma, \mathbf{K}}^{\backslash}(z, \omega) c_{\sigma, \mathbf{K}}^{\backslash}(z, \omega)\right],
\end{aligned}
$$

where we have defined the rightward and leftward operators inside the dielectric as

$$
\begin{aligned}
c_{\sigma, \mathbf{K}}^{\rangle}(z, \omega)= & a_{\sigma, \mathbf{K}}^{\rangle}(\omega) \\
& +\frac{\mu_{0}}{W_{\sigma, \mathbf{K}} \alpha_{\mathbf{K}}} \int_{-\infty}^{z} \tilde{\mathbf{U}}_{\sigma, \mathbf{K}}^{\langle}\left(z^{\prime}, \omega\right) \cdot \hat{\mathbf{j}}_{\mathbf{K}}^{+}\left(z^{\prime}, \omega\right), \\
c_{\sigma, \mathbf{K}}^{\langle}(z, \omega)= & a_{\sigma, \mathbf{K}}^{\langle}(\omega) \\
& +\frac{\mu_{0}}{W_{\sigma, \mathbf{K}} \alpha_{\mathbf{K}}} \int_{z}^{+\infty} \tilde{\mathbf{U}}_{\sigma, \mathbf{K}}^{\rangle}\left(z^{\prime}, \omega\right) \cdot \hat{\mathbf{j}}_{\mathbf{K}}^{+}\left(z^{\prime}, \omega\right),
\end{aligned}
$$

respectively. These operators become position independent and reduce simply to the photon operators $a_{\sigma, \mathbf{K}}^{\gamma /}(\omega)$ in the absence of absorption. The first term on the righthand side of Eq. (39), in the $\mathbf{K}=0, \omega=0$ limit, can be simply understood as the electrostatic field that results if the polarization sheet is assembled, in a medium of dielectric permittivity $\epsilon$, from two sheets of charge parallel to the $(x-y)$ plane. $^{31}$

\section{COMMUTATION RELATIONS}

The commutation properties of the input-photon operators were determined in Section 5. Let us now consider the commutation properties of output operators $\hat{b}_{\sigma, \mathbf{K}}^{\tau}(\omega)$. These operators, in contrast to the input photon operators, are not independent but have been related to the input photon operators and to the reservoir noise operators. Recently ${ }^{26}$ we calculated the commutation relations involving the output photon operators' performing explicit calculations for the semi-infinite dielectric. By using an integral relation for the light modes it is possible to cal- 
culate these commutation rules for arbitrary planar structures without performing explicit calculations. It can be shown that the output operators obey the following simple Bose commutation rules:

$$
\begin{aligned}
& {\left[\hat{b}_{\sigma, \mathbf{K}}^{\tau}(\omega), \hat{b}_{\sigma^{\prime}, \mathbf{K}^{\prime}}^{\tau^{\prime} \dagger}\left(\omega^{\prime}\right)\right]=\delta_{\mathbf{K}, \mathbf{K}^{\prime}} \delta_{\sigma, \sigma^{\prime}} \delta_{\tau, \tau^{\prime}} \delta\left(\omega-\omega^{\prime}\right),} \\
& {\left[\hat{b}_{\sigma, \mathbf{K}}^{\tau}(\omega), \hat{b}_{\sigma^{\prime}, \mathbf{K}^{\prime}}^{\tau^{\prime}}\left(\omega^{\prime}\right)\right]=0 .}
\end{aligned}
$$

We point out that the quantum optical input-output relations derived in Section 5 are meaningful only if they are completed by commutation relations (42) and (43), which confirm that the output operators are true photon operators that obey the Boson commutation relations prescribed for photon operators. The derivation of Eq. (43) is straightforward. Here we show the main steps in the derivation of Eq. (42); using Eqs. (35), (28), and (9), we obtain

$$
\begin{aligned}
{\left[\hat{b}_{\sigma, \mathbf{K}}^{\langle}(\omega), \hat{b}_{\sigma^{\prime}, \mathbf{K}^{\prime}}^{\langle, \dagger}\left(\omega^{\prime}\right)\right]=} & \delta\left(\omega-\omega^{\prime}\right) \delta_{\mathbf{K}, \mathbf{K}^{\prime}} \delta_{\sigma, \sigma^{\prime}} \\
& \times\left[\left|r_{\sigma}^{\rangle}\right|^{2}+\left|t_{\sigma}\right|^{2}+A_{\mathbf{K}}^{\rangle}(\omega),\right. \\
{\left[\hat{b}_{\sigma, \mathbf{K}}^{\rangle}(\omega), \hat{b}_{\sigma^{\prime}, \mathbf{K}^{\prime}}^{\rangle, \dagger}\left(\omega^{\prime}\right)\right]=} & \delta\left(\omega-\omega^{\prime}\right) \delta_{\mathbf{K}, \mathbf{K}^{\prime}} \delta_{\sigma, \sigma^{\prime}} \\
& \times\left[\left|r_{\sigma}^{\langle}\right|^{2}+\left|t_{\sigma}\right|^{2}+A_{\mathbf{K}}^{\langle\langle}(\omega),\right. \\
{\left[\hat{b}_{\sigma, \mathbf{K}}^{\rangle}(\omega), \hat{b}_{\sigma^{\prime}, \mathbf{K}^{\langle, \dagger}}^{\left\langle, \omega^{\prime}\right)=}\right.} & \delta\left(\omega-\omega^{\prime}\right) \delta_{\mathbf{K}, \mathbf{K}^{\prime}} \delta_{\sigma, \sigma^{\prime}} \\
& \times\left[r_{\sigma}^{\langle} t_{\sigma}^{*}+r_{\sigma}^{\rangle *} t_{\sigma}+A_{\mathbf{K}}^{\rangle\langle(\omega),}\right.
\end{aligned}
$$

with

$$
\begin{aligned}
& A_{\mathbf{K}}^{\tau, \tau^{\prime}}(\omega) \\
& \quad=\frac{\omega^{2}}{w_{1} c^{2}} \sum_{j} \int_{-\infty}^{\infty} \mathrm{d} z^{\prime} \epsilon_{I}\left(z^{\prime}, \omega\right) U_{j, \mathbf{K}}^{\tau}\left(z^{\prime}, \omega\right) U_{j, \mathbf{K}}^{\tau^{\prime}}\left(z^{\prime}, \omega\right) .
\end{aligned}
$$

Integrals $A_{\mathbf{K}}^{\tau, \tau^{\prime}}(\omega)$ can be evaluated by use of the following integral relation for the light modes:

$$
\begin{aligned}
\frac{\omega^{2}}{c^{2}} \int_{-L}^{L} \mathrm{~d} z \epsilon_{I}(z, \omega) & U_{\mathbf{K}, i}^{\tau}(z, \omega) U_{\mathbf{K}, j}^{\tau^{\prime} *}(z, \omega) \\
= & \frac{i}{2}\left[U_{\mathbf{K}, j}^{\tau^{\prime} *}(z, \omega) \frac{\partial}{\partial z} U_{\mathbf{K}, \sigma}^{\tau}(z, \omega)\right. \\
& \left.-U_{\mathbf{K}, i}^{\tau}(z, \omega) \frac{\partial}{\partial z} U_{\mathbf{K}, j}^{\tau^{\prime} *}(z, \omega)\right]_{-L}^{L},
\end{aligned}
$$

with $-L$ and $L$ outside the dielectric medium. Writing explicitly the surface term according to Eq. (18), and inserting it into Eqs. (44)-(46), we can readily obtain commutation relation (42) and (43).

The commutators between input and output operators can easily be calculated. Using Eqs. (35) and (28), we obtain

$$
\begin{aligned}
{\left[\hat{b}_{\sigma, \mathbf{K}}^{\rangle}(\omega), \hat{a}_{\sigma^{\prime}, \mathbf{K}^{\prime}}^{\dagger \dagger}\left(\omega^{\prime}\right)\right] } & =\left[\hat{b}_{\sigma, \mathbf{K}}^{\langle}(\omega), \hat{\mathbf{a}}_{\sigma^{\prime}, \mathbf{K}^{\prime}}^{\langle\dagger}\left(\omega^{\prime}\right)\right] \\
& =t_{\sigma} \delta_{\mathbf{K}, \mathbf{K}^{\prime}} \delta_{\sigma, \sigma^{\prime}} \delta\left(\omega-\omega^{\prime}\right), \\
{\left[\hat{b}_{\sigma, \mathbf{K}}^{(\langle,\rangle)}(\omega), \hat{a}_{\sigma^{\prime}, \mathbf{K}^{\prime}}^{\rangle,\langle\dagger}\left(\omega^{\prime}\right)\right] } & =r_{\sigma}^{\rangle /\langle} \delta_{\mathbf{K}, \mathbf{K}^{\prime}} \delta_{\sigma, \sigma^{\prime}} \delta\left(\omega-\omega^{\prime}\right) .
\end{aligned}
$$

\section{CONCLUSIONS}

We have presented a quantization scheme for the electromagnetic field in dispersive and lossy planar dielectrics that incorporates propagation in all the spatial directions and includes both TE and TM polarized fields. We have given expressions for the electric-field operator in an arbitrary planar dielectric medium, taking into account explicitly the finite extent of the medium. The electric-field operator can be expanded in frequency components given by Eq. (32) with Eq. (33). These compact expressions are valid both in free space and in the medium and include contributions from the noise operators associated with the dielectric loss mechanisms and also from the free fields incoming from free space. By writing the general Green dyadic for a planar dielectric structure in terms of the light modes, we have shown that the three-dimensional electric-field operator in free space can be written in terms of scalar photon operators in the simple form of Eq. (34), where the output operators $\hat{b}_{\sigma, \mathbf{K}}(\omega)$ are related by an input-output relation to the input photon operators $\hat{a}_{\sigma, \mathbf{K}}(\omega)$ and to reservoir noise operators $\hat{\mathbf{f}}_{\mathbf{K}}(z, \omega)$. These three-dimensional input-output relations for arbitrary planar dielectrics represent the most important result of this paper. The expressions obtained for the output photon operator are easy to use in practical calculations; they require only knowledge of the classic light modes, and they can readily be applied to analysis of input-output problems. We point out that it has been possible to define output operators that are scalars, although they depend on the noise operators, which are vectors. By using a set of integral relations for the light modes [Eq. (48)], we have verified that the defined output operators satisfy the prescribed Boson commutation relations.

O. Di Stefano's e-mail address is distef@ortica.unime.it; that of R. Girlanda is raffaello.girlanda@unime.it.

\section{REFERENCES}

1. E. M. Purcell, "Spontaneous emission probabilities of radio frequencies," Phys. Rev. 69, 681 (1946).

2. G. S. Agarwal, "Coherence in spontaneous emission in the presence of a dielectric," Phys. Rev. Lett. 32, 703-706 (1974).

3. E. Yablonovitch, "Inhibited spontaneous emission in solidstate physics and electronics," Phys. Rev. Lett. 58, 20592062 (1987).

4. S. M. Barnett, B. Huttner, and R. Loudon, "Spontaneous emission in absorbing dielectric media," Phys. Rev. Lett. 68, 3698-3701 (1992).

5. S. Scheel, L. Knöll, D.-G. Welsch, and S. M. Barnett, "Quantum local-field corrections and spontaneous decay," Phys. Rev. A 60, 1590-1597 (1999).

6. Milloni, The Quantum Vacuum: An Introduction to Quantum Electrodynamics (Academic, San Diego, Calif., 1994).

7. M. Artoni and R. Loudon, "Propagation of nonclassical light through an absorbing and dispersive slab," Phys. Rev. A 59, 2279-2290 (1999). 
8. E. Schmidt, L. Knöll, and D.-G. Welsch, "Propagation of squeezed-light pulses in dispersive and absorbing linear dielectrics," Phys. Rev. A 54, 843-855 (1996).

9. L. Knöll, E. Schmidt, D.-G. Welsh, and A. V. Chizhov, "Quantum-state transformation by dispersive and absorbing four-port devices," Phys. Rev. A 59, 4716-4726 (1999).

10. C. H. Henry and F. Kazarinov, "Quantum noise in photonics," Rev. Mod. Phys. 68, 801-853 (1996).

11. B. Huttner and S. M. Barnett, "Quantization of the electromagnetic field in dielectrics," Phys. Rev. A 46, 4306-4322 (1992).

12. L. Knöll and U. Leonhardt, "Quantum optics in oscillator media," J. Mod. Opt. 39, 1253 (1992).

13. R. Matloob, R. Loudon, S. M. Barnett, and J. Jeffers, "Electromagnetic field quantization in absorbing dielectrics," Phys. Rev. A 52, 4823-4838 (1995).

14. R. Matloob and R. Loudon, "Electromagnetic field quantization in absorbing dielectrics. II," Phys. Rev. A 53, 45674582 (1996).

15. T. Gruner and D. G. Welsh, "Green-function approach to the radiation-field quantization for homogeneous and inhomogeneous Kramers-Kronig dielectrics," Phys. Rev. A 53, 1818-1829 (1996)

16. U. Leonhardt, "Influence of a dispersive and dissipative medium on spectral squeezing," J. Mod. Opt. 40, 1123-1130 (1993).

17. D. Kupiszewska, "Casimir effect in absorbing media," Phys. Rev. A 46, 2286-2294 (1992).

18. M. Artoni and R. Loudon, "Quantum theory of optical pulse propagation through an absorbing and dispersive slab," Phys. Rev. A 55, 1347-1357 (1997).

19. T. Gruner and D. G. Welsch, "Quantum-optical inputoutput relations for dispersive and lossy multilayer dielectric plates," Phys. Rev. A 54, 1661-1677 (1996).

20. O. Di Stefano, S. Savasta, and R. Girlanda, "Mode expan- sion and photon operators in dispersive and absorbing dielectrics," J. Mod. Opt. 48, 67-84 (2001).

21. O. Di Stefano, S. Savasta, and R. Girlanda, "Electromagnetic-field quantization in absorbing confined systems," Phys. Rev. A 60, 1614-1625 (1999).

22. H. T. Dung, L. Knöll, and D. G. Welsh, "Three-dimensional quantization of the electromagnetic field in dispersive and absorbing inhomogeneous dielectrics," Phys. Rev. A 57, 3931-3942 (1998).

23. S. Scheel, L. Knöll, and D.-G. Welsch, "QED commutation relations for inhomogeneous Kramers-Kronig dielectrics," Phys. Rev. A 58, 700-706 (1998).

24. A. Tip, "Linear absorptive dielectrics," Phys. Rev. A $\mathbf{5 7}$ 4818-4841 (1998)

25. A. Tip, L. Knöll, S. Scheel, and D.-G. Welsch, "Equivalence of the Langevin and auxiliary-field quantization methods for absorbing dielectrics," Phys. Rev. A 63, 043806 (7 pages) (2001).

26. O. Di Stefano, S. Savasta, and R. Girlanda, "Threedimensional electromagnetic field quantization in absorbing and dispersive bounded dielectrics," Phys. Rev. A 61, 023803 (12 pages) (2000).

27. H. A. Mcleod, Thin Film Optical Filters (Elsevier, New York, 1969)

28. P. Yeh, Optical Waves in Layered Media (Wiley, New York, 1988).

29. M. S. Tomaš, "Green function for multilayers: Light scattering in planar cavities," Phys. Rev. A 51, 2545-2559 (1995).

30. D. L. Mills and A. A. Maradudin, "Surface roughness and the optical properties of a semi-infinite material: the effect of a dielectric overlayer," Phys. Rev. B 12, 2943-2958 (1975).

31. J. E. Sipe, "New Green-function formalism for surface optics," J. Opt. Soc. Am. B 4, 481-489 (1987). 